\title{
A Communication Theoretic Analysis of Synaptic Channels under Axonal Noise
}

\author{
Behrouz Maham, Senior Member, IEEE
}

\begin{abstract}
Molecular communication is an emerging communication technology for applications requiring nanoscale networks. Transferring vital information about external and internal conditions of the body through the nervous system is an important type of intra-body molecular nanonetworks. Thus, investigating the performance of such systems from the communication theoretic perspective gives us insight on the limitation of neuro-spike communication and ways to design artificial neural systems. In this letter, we study the performance of the neuro-spike communication under different stochastic impairments such as axonal shot noise, synaptic noise, and random vesicle release. The objective is to optimally detect the spikes at the receiving neuron. Since several uncertainties occur under each hypothesis, composite hypothesis is employed to find the optimum detection policy. Furthermore, we obtain closed-form solutions for the optimal detector and derive the binary decision error at the postsynaptic terminal.
\end{abstract}

\section{INTRODUCTION}

The molecular communications has recently received a lot of attention in the area of communication theory due to its capability in nano-scale communications (see, e.g., [1]-[3]). A widely used molecular communication in biological systems is the nervous system which is responsible for the transmission of vital information through the body and brain. Although electric signals are used for carrying information in axons, adjacent neurons are connected by molecular communications. The latter is called neuro-spike communication and occurs at the synaptic terminals. For example, a hybrid (molecular and electromagnetic) communication paradigm is investigated in [4] to characterize the signal propagation in neuronal systems. To be able to have a long-range communication and to excite the release of molecules at the synapses, a wide-band spikelike waveform is used in the nervous system.

Most of the existing work on the encoding and decoding of neural systems has focused on the problem of predicting neural responses to estimate the stimuli, and thus, the rest of the synaptic communication system is observed as a black-box [5]. However, in this work, similar to [6], [7], we consider the mathematical model of neuro-spike transmission through the central synapses and investigate the detection of spikes at the receiving neurons. The neuro-spike communication consists of two main noise types, i.e., axonal noise and synaptic noise. In this letter, in contrast to [6], [7], we take both of these noise sources into account. As stated in [8], the axonal noise is more prominent in thin axons of less than $1 \mathrm{~mm}$ of diameter. Examples consist of C-fibres in sensory and pain transmission, cerebellar parallel fibres, and cortical pyramidal cell axon collaterals that form most of the local cortical connectivity [8].

Behrouz Maham is with the Department of Electrical and Electronic Engineering, School of Engineering, Nazarbayev University, Astana, Kazakhstan. Email: behrouz.mahamenu.edu.kz.
In this letter, we consider a point-to-point communication between two neurons at the central synapses which consists of several sources of randomness including axonal noise, random vesicle release, random amplitude, and synaptic noise. We investigate the performance of the neuro-spike transmission and detection under the mentioned communication channel. Due to the refractory effect [5], the inter-spike interval cannot be less than a certain time. Thus, the synaptic signal can be modeled by a binary random process, in which zero and one mean the events of no-spike and one-spike, respectively. The optimum binary detector is found. Since several uncertainties occur under each hypothesis, composite hypothesis is employed to find the optimum spike detection. Furthermore, the closedform solution for the likelihood-ratio is derived. Moreover, the probability of error at the receiving neuron is investigated. In numerical results, we show that impairments like axonal noise should be considered in the mathematical modeling of neuro-spike communications.

\section{Channel Model of a Neuro-Spike System}

As shown in Fig. 1, we consider a mathematical model for the neuro-spike communication channel between the presynaptic neuron and postsynaptic neuron in central synapses, e.g., the synapse in the hippocampus of cerebral cortex. The spike train is generated from the stimuli through the process of neural spike response (see, e.g., [9]). Thus, the generated spike train in the axon can be represented as $s(t)=\sum_{k} \delta\left(t-t_{k}\right)$, where $\delta(\cdot)$ is a Dirac delta function and $t_{k}$ is the time that a spike occurs. A spike is generated by a rapid change on the membrane potential and is also called action potential (AP). The duration of AP is about $1 \mathrm{~ms}$ with roughly $100 \mathrm{mV}$ electric voltage fluctuation [5, p. 4], and thus, it can be well modeled by a delta function.

The continuous time in $s(t)$ can be split into time slots of size $\tau_{f}$. The time slots are chosen small enough such that no more than one impulse occurs in a bin. Note that this assumption is quite realistic since as a result of the relative refractory effect (see, e.g., [5, p. 32]), short inter-spike intervals become less likely than the one Poisson model predicts. Thus, the interval between consecutive impulses usually gets larger than $\tau_{f}$, and the occurrence of two impulses in a time slot of size $\tau_{f}$ becomes almost impossible. Each time slot is labeled as $X=0$ when no spike occurred and as $X=1$ when there is a spike in the bin. It is shown in Fig. 1 that the spike train can be modeled by passing the binary input through the binary encoding or on-off-keying modulation with delta-like pulses.

The axonal noise is caused by the random opening of ion channels, which is independent of spike train $s(t)$. Similar to 


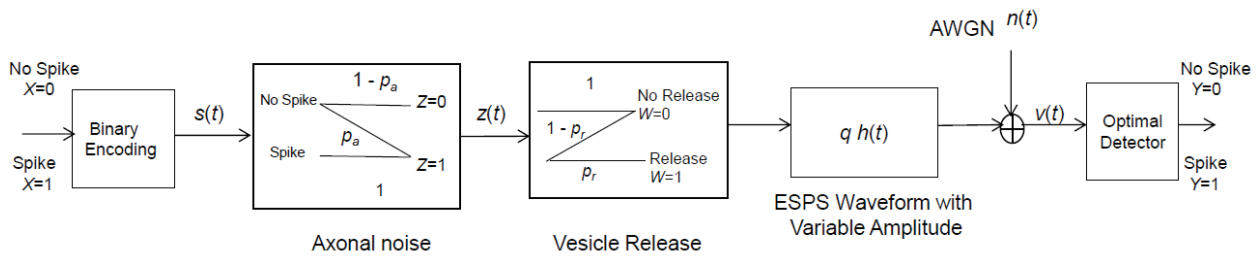

Fig. 1. The mathematical model for the neuro-spike communication channel.

[7], we model the axonal noise as a binary stochastic process. That is, in time $t$, one erroneous spike due to the axonal noise occurs with probability of $p_{a}$, and with probability of $1-p_{a}$, there is no erroneous spike. The spike train exposed to axonal noise is called $z(t)$. It is shown in Fig. 1 that the output of axonal noise block at time $t$ can be represented by a binary random variable $Z$. The event $Z=1$ means that there is one spike in a time slot with probability of $\operatorname{Pr}\{Z=1\}=\operatorname{Pr}\{X=$ $1\}+p_{a} \operatorname{Pr}\{X=0\}=1-p_{0}+p_{a} p_{0}$. where $p_{0}=\operatorname{Pr}\{X=$ $0\}$ is the prior probability of spike absence. In addition, the event of no spike in $z(t)$ in the range between $t$ and $t+\tau_{f}$ can be represented by $Z=0$ and its probability is given by $\operatorname{Pr}\{Z=0\}=\left(1-p_{a}\right) \operatorname{Pr}\{X=0\}=p_{0}-p_{a} p_{0}$.

When an AP reaches the synapse, its voltage transient causes the release of neurotransmitters from at most one of vesicles residing at the presynaptic side of a neuron [5]. The vesicles inside the presynaptic terminal contain neurotransmitters. The neurotransmitters are transmitted with probability $p_{r}$ which is the vesicle release probability. This mechanism is shown in Fig. 1 and is modeled by a binary $\mathrm{Z}$ channel with probability of $p_{r}$. Note that, in response to an AP, the probability of vesicle release could be quite low. For example, $p_{r}$ could be as low as 0.1 (see [10] for the exact amount of $p_{r}$ in different types of synapses).

Besides axonal noise and vesicle release process, there is another form of uncertainty in the mathematical model of synaptic transmission. This uncertainty is due to the variable postsynaptic response to vesicle release and is called variable quantal amplitude denoted by $q$. The influx of neurotransmitters from the presynaptic terminal is absorbed by receptors at the postsynaptic terminal of the receiving neuron. This part of the channel is diffusion-based molecular communication. The diffused neurotransmitters bind to the receptors at the postsynaptic terminal. By opening of the receptors, excitatory postsynaptic current (EPSC) are driven into the receiving neuron to produce an AP there. The induced potential across the membrane of the receiving neuron is called excitatory postsynaptic potential (EPSP). The EPSP amplitude distribution is optimally fitted with a $k$ th-order Gamma distribution [11]

$$
f_{q}(q)=\frac{\lambda^{k}}{(k-1) !} q^{k-1} \exp (-\lambda q),
$$

where $\lambda=\mu / \sigma^{2}, k=\mu^{2} / \sigma^{2}$, and $\mu$ and $\sigma^{2}$ are the mean and variance of the random amplitude $q$ of the postsynaptic response, respectively. Moreover, the EPSP waveform can be represented by an $\alpha$ function as [6]

$$
h(t)=\frac{h_{\max } t}{\tau_{\max }} \exp \left(1-t / \tau_{\max }\right) u(t),
$$

where $h_{\max }$ and $\tau_{\max }$ denote the peak point of the waveform and its corresponding time-to-peak, respectively, and $u(t)=1$ for $t>0$ and 0 otherwise. Note that $h(t)$ gets close to $\delta(t)$ asymptotically as $h_{\max } \rightarrow \infty$ and $\tau_{\max } \rightarrow 0$. The value of $h_{\max }$ and $\tau_{\max }$ depend on the type of receptors (e.g., AMPA, NMDA, GABA, etc) [5].

Finally, in a time slot of length $\tau_{f}$, i.e., $t \in\left[t_{0}, t_{0}+\tau_{f}\right)$, where $t_{0}$ is the start of a time slot in which at most one spike may occur, the postsynaptic membrane voltage becomes $v(t)=W q h(t)+n(t)$, where $n(t)$ is the synaptic background white noise with variance $\sigma_{n}^{2}$ which has a Gaussian distribution due to central limit theorem [7]. In $v(t), W$ is a Bernoulli random variable representing the vesicle release process, where the event $W=1$ stands for the vesicle release from the presynaptic terminal, and $W=0$ otherwise.

\section{Analysis of the Signal Detection}

In this section, based on the observation of the received postsynaptic signal $v(t)$, we recover the binary information $X$ and calculate the probability of binary decision error.

It is well known that for minimizing the error of binary transmission in additive white Gaussian noise (AWGN), the matched filter can be used. Equivalently, the correlation between the received signal and the EPSP response $h(t)$ can be calculated to make the optimal binary decision.

Now, formulating the problem as a statistical hypothesis test, we try to find the probability of error in recovering the transmitted binary random variable $X$. We assume that the received postsynaptic signal $v(t)$ is observable in a single time slot of length $\tau_{f}$. The hypothesis test for each spike decision can be represented as

$$
\left\{\begin{array}{l}
\mathrm{H}_{0}: v(t)=n(t), \\
\mathrm{H}_{1}: v(t)=W q h(t)+n(t),
\end{array}\right.
$$

where $\mathrm{H}_{0}$ and $\mathrm{H}_{1}$ refer to the hypotheses of spike absence $(X=0)$ and spike presence $(X=1)$, respectively. The choice of $\tau_{f}$ should be long enough for one spike only. Because of the randomness in $n(t), W$, and $q$, the outcomes of $\mathrm{H}_{0}$ and $\mathrm{H}_{1}$ are stochastic processes.

By defining $\underline{v}=\left[v\left(t_{1}\right), v\left(t_{2}\right), \ldots, v\left(t_{n}\right)\right]$ in the interval of length $\tau_{f}$, the $n$-dimensional joint probability density function (PDF) of $v(t)$ conditioned on $\mathrm{H}_{0}$ and $\mathrm{H}_{1}$ are given by $f_{V}\{\underline{v} \mid X=0\}$ and $f_{V}\{\underline{v} \mid X=1\}$, respectively.

By applying the Bayes criterion [12], the likelihood ratio for the hypothesis test in (3) is defined as

$$
L_{X}(v)=\frac{f_{V}\{\underline{v} \mid X=1\}}{f_{V}\{\underline{v} \mid X=0\}} .
$$

For the binary decision, the region $\mathrm{H}_{0}$ consists of values of $\underline{v}$ for which $L_{X}(v)<L_{0}$, and $\mathrm{H}_{1}$ of values of $\underline{v}$ for which 
$L_{X}(v)>L_{0}$. The critical threshold $L_{0}$ is given by $L_{0}=$ $p_{0} /\left(1-p_{0}\right)$.

Since $W$ in $\mathrm{H}_{1}$ is also a random variable, in the following, we try to write the likelihood ratio in (4) in terms of

$$
L_{W}(v)=\frac{f_{V}\{\underline{v} \mid W=1\}}{f_{V}\{\underline{v} \mid W=0\}} .
$$

Using the law of total probability, we have

$$
\begin{aligned}
f_{V}\{\underline{v} \mid X=0\}= & f_{V}\{\underline{v} \mid W=0, X=0\} \operatorname{Pr}\{W=0 \mid X=0\} \\
& +f_{V}\{\underline{v} \mid W=1, X=0\} \operatorname{Pr}\{W=1 \mid X=0\} \\
= & f_{V}\{\underline{v} \mid W=0\} \operatorname{Pr}\{W=0 \mid X=0\} \\
& +f_{V}\{\underline{v} \mid W=1\} \operatorname{Pr}\{W=1 \mid X=0\} .
\end{aligned}
$$

By using the marginal probability and chain rules, the conditional probabilities in (6) can be represented in terms of axonal noise probability $p_{a}$ as:

$$
\begin{aligned}
\operatorname{Pr}\{W=0 \mid X=0\}= & \operatorname{Pr}\{W=0, Z=0 \mid X=0\} \\
& +\operatorname{Pr}\{W=0, Z=1 \mid X=0\} \\
= & \operatorname{Pr}\{W=0 \mid Z=0\} \operatorname{Pr}\{Z=0 \mid X=0\} \\
& +\operatorname{Pr}\{W=0 \mid Z=1\} \operatorname{Pr}\{Z=1 \mid X=0\} \\
= & \left(1-p_{a}\right)+\left(1-p_{r}\right) p_{a}=1-p_{a} p_{r},
\end{aligned}
$$

where in the second equality, we used the facts that $\operatorname{Pr}\{W=0 \mid X=0, Z=0\}=\operatorname{Pr}\{W=0 \mid Z=0\}$ and $\operatorname{Pr}\{W=0 \mid X=0, Z=1\}=\operatorname{Pr}\{W=0 \mid Z=1\}$. Similarly, we have $\operatorname{Pr}\{W=1 \mid X=0\}=p_{a} p_{r}$. Hence, the conditional $\mathrm{PDF}$ in (6) can be rewritten as

$f_{V}\{\underline{v} \mid X=0\}=f_{V}\{\underline{v} \mid W=0\}\left(1-p_{a} p_{r}\right)+f_{V}\{\underline{v} \mid W=1\} p_{a} p_{r}$

Similarly, it can be shown that

$f_{V}\{\underline{v} \mid X=1\}=\left(1-p_{r}\right) f_{V}\{\underline{v} \mid W=0\}+p_{r} f_{V}\{\underline{v} \mid W=1\}$.

Combining (4), (5), (8), and (9), the likelihood ratio $L_{X}(v)$ is expressed as

$$
L_{X}(v)=\frac{p_{r} L_{W}(v)+1-p_{r}}{1-p_{a} p_{r}+p_{a} p_{r} L_{W}(v)} .
$$

Thus, the binary decision can be expressed in terms of $L_{W}(v)$ as

$$
L_{W}(v) \underset{\mathrm{H}_{0}}{\stackrel{\mathrm{H}_{1}}{\gtrless}} \frac{\left(1-p_{a} p_{r}\right) L_{0}-1+p_{r}}{p_{r}-p_{a} p_{r} L_{0}} \triangleq L_{1} .
$$

Thus, as shown in Fig. 1, the decision rule in (11) can be used as the optimum detector at the receiving neuron.

Since the amplitude $q$ of the transmitted signal under $\mathrm{H}_{1}$ is random, we employ the composite hypothesis testing used in detection of signals with unknown parameters [12]. Conditioned on $q, \underline{v}$ has jointly Gaussian distribution under both hypotheses. Thus, from (5) and by using the CameronMartin formula for signal detection in continuous time [12], when $n \rightarrow \infty$, we have

$$
L_{W}(v \mid q)=\exp \left\{\frac{2 q \int_{0}^{\tau_{f}} h(t) v(t) d t-q^{2} E_{h}}{N_{0}}\right\},
$$

where $N_{0}$ is the power spectral density of $n(t)$ and $E_{h}=$ $\int_{0}^{\tau_{f}} h^{2}(t) d t$ is the energy of the EPSP response $h(t)$. To avoid inter-symbol interference, $h(t)$ should be zero outside the support interval of length $\tau_{f}$, i.e., $h(t) \cong 0$ for $t>\tau_{f}$. Thus, from (2), we have

$$
E_{h} \cong \frac{h_{\max }^{2} e^{2}}{\tau_{\max }^{2}} \int_{0}^{+\infty} t^{2} e^{\frac{-2 t}{\tau_{\max }}} d t=\frac{e^{2}}{4} \tau_{\max } h_{\max }^{2} .
$$

Next, in the composite hypothesis testing, the conditional likelihood ratio should be averaged over unknown parameter as

$$
\begin{aligned}
L_{W}(v) & =\int_{0}^{\infty} f_{q}(q) \exp \left\{\frac{2 q r(v)-q^{2} E_{h}}{N_{0}}\right\} d q \\
& =\int_{0}^{\infty} \frac{\lambda^{k} q^{k-1}}{(k-1) !} e^{-\lambda q} e^{\frac{2 q r(v)-q^{2} E_{h}}{N_{0}}} d q,
\end{aligned}
$$

where $r(v)=\int_{0}^{\tau_{f}} h(t) v(t) d t$ is the correlation between the received signal and EPSP waveform $h(t)$.

From [13, Eq. 3.462], the closed-form solution for the integral in (14) can be obtained as

$$
\begin{aligned}
L_{W}(v)= & \lambda^{k}\left(\frac{2 E_{h}}{N_{0}}\right)^{-k / 2} \exp \left[\frac{\left(\lambda N_{0}-2 r(v)\right)^{2}}{8 N_{0} E_{h}}\right] \\
& \times D_{-k}\left(\frac{\lambda N_{0}-2 r(v)}{\sqrt{2 N_{0} E_{h}}}\right)
\end{aligned}
$$

where $D_{p}(\cdot)$ is the parabolic cylinder function of order $p[13$, Eq. 9.240]. Hence, we obtained a closed-form solution for the optimal detector.

The parameter $k$ in the amplitude distribution of $f_{q}(q)$ in (1) is used to model the variability of $q$ [6]. For the case of $k=1$, the gamma distribution has the highest variability or the worst-case scenario, and by using [13, Eq. 3.322], $L_{W}(v)$ becomes

$$
L_{W}(v)=\lambda \sqrt{\frac{N_{0} \pi}{E_{h}}} e^{\frac{\left(\lambda N_{0}-2 r(v)\right)^{2}}{4 N_{0} E_{h}}} Q\left(\frac{\lambda N_{0}-2 r(v)}{2 \sqrt{N_{0} E_{h}}}\right)
$$

where $Q(x)=1 / \sqrt{2 \pi} \int_{x}^{\infty} \exp \left(-u^{2} / 2\right) d u$ is the q-function or the tail probability of the standard normal distribution.

Next, we calculate the error in detecting the transmitted spikes. From the law of total probability, the average probability of error incurred by the decision rule in (11) is given by

$$
P_{e}=p_{0} P_{F}+\left(1-p_{0}\right) P_{M} \text {. }
$$

The probability $P_{F}$ of selecting hypothesis $\mathrm{H}_{1}$ when $\mathrm{H}_{0}$ is correct is called false detection probability and is calculated as

$$
P_{F}=\operatorname{Pr}\{Y=1 \mid X=0\}=\operatorname{Pr}\left\{L_{W}(v)>L_{1} \mid X=0\right\}
$$

where $Y=1$ denoted the event that spike is detected at the optimal detector output. In addition, the probability $P_{M}$ of selecting hypothesis $\mathrm{H}_{0}$ when $\mathrm{H}_{1}$ is correct is called missdetection probability and is calculated as

$$
P_{M}=\operatorname{Pr}\{Y=0 \mid X=1\}=\operatorname{Pr}\left\{L_{W}(v) \leq L_{1} \mid X=1\right\}
$$

where $Y=0$ denoted the event that spike is not detected at the optimal detector output. Then, similar to the procedure to obtain (8) and (9), $P_{F}$ and $P_{M}$ can be simplified to

$$
\begin{aligned}
P_{F}= & p_{a} p_{r} \operatorname{Pr}\left\{L_{W}(v)>L_{1} \mid W=1\right\} \\
& +\left(1-p_{a} p_{r}\right) \operatorname{Pr}\left\{L_{W}(v)>L_{1} \mid W=0\right\},
\end{aligned}
$$




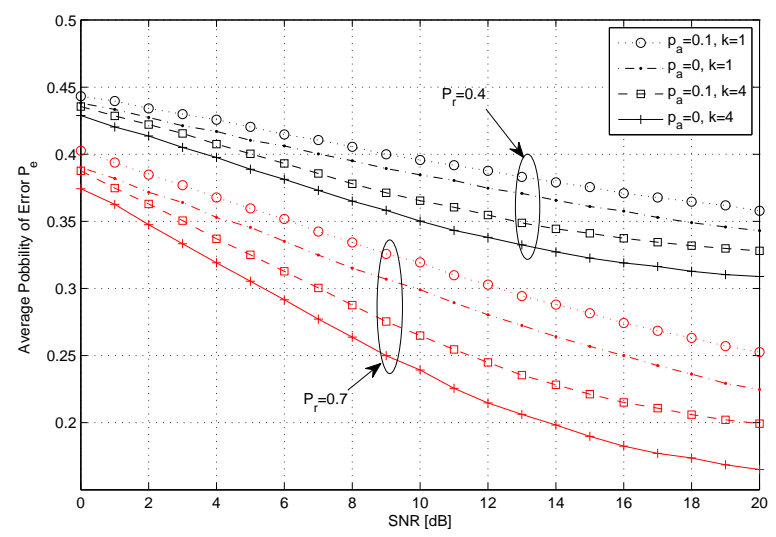

Fig. 2. The average detection error probability $P_{e}$ curves versus the signalto-noise ratio for different parameters of axonal noise, vesicle release, and random amplitude $q$ in a neuro-spike communication channel.

$$
\begin{aligned}
P_{M}= & \left(1-p_{r}\right) \operatorname{Pr}\left\{L_{W}(v) \leq L_{1} \mid W=0\right\} \\
& +p_{r} \operatorname{Pr}\left\{L_{W}(v) \leq L_{1} \mid W=1\right\} .
\end{aligned}
$$

The likelihood ratio $L_{W}(v)$ in (15) is a function of random variable $r(v)$, and thus, the CDF of $L_{W}(v)$ can be expressed in terms of the CDF of $r(v)$. If $W=0$, we have $r(v)=$ $\int_{0}^{\tau_{f}} h(t) v(t) d t=n_{\text {out }}$, where $n_{\text {out }}$ is again a white Gaussian noise. In addition, if $W=1$, we have $r(v)=E_{h} q+n_{\text {out }}$, which is the summation of gamma and zero-mean Gaussian random variables.

\section{NUMERICAL RESULTS}

In this section, the numerical results are performed to illustrate the performances of the detection scheme presented in the previous section. The elements of channel amplitude are generated by the Gamma distribution model with the mean $\mu=1$ and $k=1,4$. Similar to [6], we assume the EPSP waveform with $h_{\max }=2 \mathrm{mV}$ and $\tau_{\max }=1 \mathrm{msec}$. The refractory period can be chosen as a Gaussian random variable with mean $5 \mathrm{msec}$ [5]. Thus, we choose $\tau_{f}=5 \mathrm{msec}$.

In Fig. 2, the performance of binary neuro-spike communication system under different stochastic impairments are examined. The curves are obtained by averaging the false alarm and miss-detection error events over 100000 transmitted spikes. The average detection error curves versus SNR, i.e., $E_{h} / N_{0}$, at the postsynaptic neuron are shown for different values of axonal shot noise parameter $p_{a}$, synaptic release probability $p_{r}$, and the variable quantal amplitude with Gamma distribution parameter $k$. It is shown that at $P_{e}=0.2$, around $6 \mathrm{~dB}$ more SNR required when there is axonal noise with $p_{a}=0.1$, compared to a system with no axonal noise, in a channel with $p_{r}=0.7$ and $k=4$. It is also shown that at high SNR conditions, there is an error-floor caused by random vesicle release and axonal shot noise. In addition, one can observe that by increasing the parameter $k$, the performance is improved due to the reduction in channel amplitude variability. In Fig. 3, the average probability of error is shown versus the axonal noise parameter $p_{a}$ for different vesicle release probabilities $p_{r}=0.3,0.5,0.7,0.9$, when $\mathrm{SNR}=10 \mathrm{~dB}$ and $k=1$. In all cases, the performance is degraded considerably for $p_{a}>0.1$.

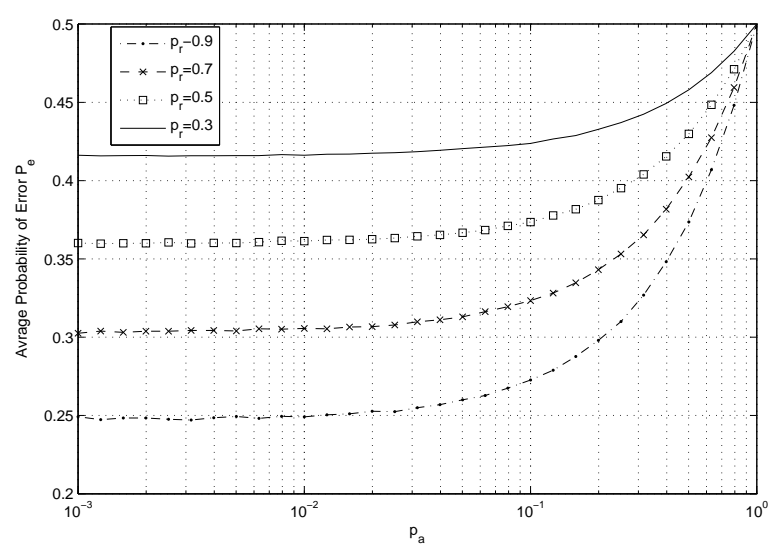

Fig. 3. The average detection error probability $P_{e}$ curves versus the axonal noise parameter $p_{a}$ for different vesicle release probabilities $p_{r}$ in a channel with $\mathrm{SNR}=10 \mathrm{~dB}$ and $k=1$.

\section{CONCLUSION}

In this paper, we have studied the performance of the neurospike transmission and detection under the communication channel consisting of several sources of randomness including axonal noise, random vesicle release, random amplitude, and synaptic noise. The optimum binary detector was found. Furthermore, the probability of error at the receiving neuron was derived. It was also shown that impairments like axonal noise which is prominent in thin axons should be considered in the mathematical modeling of neuro-spike communications.

\section{REFERENCES}

[1] I. F. Akyildiz, F. Brunetti, and C. Blázquez, "Nanonetworks: a new communication paradigm," Computer Networks, vol. 52, no. 6, pp. 22602279, Aug. 2008.

[2] L. Galluccio, S. Palazzo, and G. E. Santagati, "Characterization of molecular communications among implantable biomedical neuroinspired nanodevices," Nano Communication Networks, vol. 4, no. 2, pp. 53-64, Jun. 2013.

[3] N. Farsad, N.-R. Kim, A. W. Eckford, and C.-B. Chae, "Channel and noise models for nonlinear molecular communication systems," IEEE J. Select. Areas Commun., vol. 32, no. 12, pp. 2391-2401, Dec. 2014.

[4] L. Galluccio, S. Palazzo, and G. E. Santagati, "Characterization of signal propagation in neuronal systems for nanomachine-to-neurons communications," in Proc. of IEEE Monacom, Apr. 2011.

[5] P. Dayan and L. F. Abbott, Theoretical Neuroscience: Computational and Mathematical Modeling of Neural Systems. Massachusetts, USA: MIT Press, 2001.

[6] A. Manwani and C. Koch, "Detecting and estimating signals over noisy and unreliable synapses: information-theoretic analysis," Neural Computation, vol. 13, no. 1, pp. 1-33, Jan. 2001.

[7] E. Balevi and O. Akan, "A physical channel model for nanoscale neurospike communications," IEEE Trans. Commun., vol. 61, no. 3, pp. 1178 1187, Mar. 2013.

[8] A. Neishabouri1 and A. A. Faisal, "Axonal noise as a source of synaptic variability,” PLOS Comp. Biology, vol. 10, no. 5, pp. 1-16, May 2014.

[9] D. Malak and O. Akan, "A communication theoretical analysis of synaptic multiple-access channel in hippocampal-cortical neurons," IEEE Trans. Commun., vol. 61, no. 6, pp. 2457-2467, Jun. 2013.

[10] T. Branco and K. Staras, "The probability of neurotransmitter release: variability and feedback control at single synapses," Nature Reviews Neuroscience, vol. 10, pp. 373-383, May 2009.

[11] J. M. Bekkers and J. D. Clements, "Quantal amplitude and quantal variance of strontium-induced asynchronous EPSCs in rat dentate granule neurons," Journal of Physiology, vol. 516, no. 1, pp. 227-248, 1999.

[12] C. Helstrom, Statistical Theory of Signal Detection. Oxford, UK, 2nd edition: Pergamon Press, 1975.

[13] I. S. Gradshteyn and I. M. Ryzhik, Table of Integrals, Series, and Products. San Diego, USA: Academic, 1996. 